\title{
Lipemia in patients who receive treatment with psychopharmacos
}

\begin{abstract}
Currently the population faces high stress and anxiety, which generates the development of pathologies such as mental illness, with a prevalence of 4.3 to $26.4 \%$, accounting for $12 \%$ of global morbidity. Mental illnesses have a major impact on mortality, morbidity and disability at all stages of life, also as part of a social problem and public health problem. Pharmacological treatment of these drugs has been estimated at 6 weeks, there are cases that have the required drug combinations, reason why, it is important to take into account the types of drugs that can be used simultaneously, and Thatpsychopharmaceuticals are grouped according to their chemical characteristics and their effect on the neurotransmitter systems. Thus, the observable characteristics of pharmacodynamics are diverse for each type of drug, as well as for the various patients requiring treatment. The supplement may cause clinical manifestations such as intoxications and metabolic alterations, leading to the development of various pathologies, which may complicate the patient's condition during and after psychiatric treatment. The role of serum lipids in the pathophysiology of psychiatric disorders is unclear, can determine the availability of serotonin receptors and the decrease of their use. Patients with mental disorders under treatment are at high risk of developing dyslipidemia, by activating the transcription factors of cholesterol-regulating proteins, which is an essential constituent of myelin, which is deficient in stress conditions, Inducing the decrease in protein protein (AMPK- $\alpha$ ) and HMG-CoA reductase, which is the limiting enzyme in cholesterol synthesis and the two in cholesterol concentration in brain.
\end{abstract}

Volume 5 Issue $3-2017$

\author{
Fernanda Gutiérrez, María Brett, Marvin \\ Querales \\ Department of Biochemistry, Universidad de Carabobo, \\ Venezuela
}

Correspondence: Fernanda Gutiérrez, Bioanalysis Bachelor Degree, Magiser in Analytical Toxicology, Department of Biochemistry, Faculty of Health sciences, Universidad de Carabobo,Venezuela, Email fergutierrrrez@gmail.com

Received: June 23, 2017 | Published: July 05, 2017

Keywords: anxiety, pharmacodynamics, cholesterol, AMPK- $\alpha$, mortality, morbidity

Abbreviations: BZD, dibenzodiazepine; SSRIs, selective serotonin reuptake inhibitors; HDLC, high density lipoprotein C; SREBP, sterol regulatory element binding protein; HMG CoA REDUCTASE, hydroxymethylglutaryl CoA reductase

\section{Introduction}

At present, the human population faces innumerable episodes of stress, depression and anxiety that cause the development of pathologies, in which various modifiable and non-modifiable factors influence. With individual differences in vulnerability to stress. ${ }^{1}$ Mental disorders occur when there are problems in cognitive, emotional or perceptual functioning in a person, causing significant dysfunction in one or more areas of social or occupational performance. ${ }^{2}$ In 2006, a study was conducted on the prevalence of mental disorders in adults, reporting figures ranging from $4.3 \%$ to $26.4 \%$ depending on the country, with the United States of America (26.4\%), Ukraine (20.5\%), France (18.4\%) and Colombia (17.8\%) had the highest and lowest rates in the People's Republic of China Shanghai (4.3\%), Nigeria $(4.7 \%)$, Italy $(8.2 \%)$ and Japan $(8.8 \%))^{2}$ On the other hand, it is estimated that mental disorders account for $12 \%$ of the global burden of morbidity, but only a minority of those affected receive basic treatment. At the same time, in Latin America and the Caribbean, it accounts for $22 \%$ of the total burden of disease. It is clear that mental illness has a significant impact on mortality, morbidity and disability at all stages of life. ${ }^{3,4}$ It is verified that the organisms present dysfunction, when they present states of repeated or prolonged stress. ${ }^{1}$ Thus, it has been established that the most prevalent mental disorders are those of depression and anxiety; According to this, depression has been related to family and partner problems and may be present in a mild or accentuated way, therefore, it is considered not only as a medical problem, but also, as part of a phenomenon Social and public health, since it is observed in $3.7 \%$ of the general population. ${ }^{1,5-7}$
Previously, the treatment of depression consisted of obtaining a satisfactory response in the short term, usually 6 weeks; The popularity of antidepressants in the treatments prescribed by doctors in the different specialties, certified this priority success for the patient. However, psychiatrists observed that relapse and recurrence were the reason for consultation for a large majority of patients with disorders of depression and anxiety. ${ }^{4}$ For this reason, numerous types of psychoactive drugs have been used, stating that antidepressants are effective in approximately $60 \%$ of patients, although more than $30 \%$ respond to a placebo. ${ }^{3,7}$ There are particularly severe cases that require the combination of drugs, so it is important to take into account the types of drugs that can be used simultaneously, since anti-depressants are grouped according to their chemical characteristics and their effect on neurotransmitter systems. For example, there are non-selective and selective serotonin reuptake inhibitors (SSRIs or ISR-5HT) and noradrenaline recaptures and heterocyclics such as dibenzodiazepine (BZD). Thus, the observable characteristics of pharmacodynamics are different for each type of drug, as well as for the different patients requiring treatment. ${ }^{7}$ The processes of absorption, distribution and elimination of the drug vary over time; for this reason, the amount of drug in the organism does not remain static, but varies; On the other hand, that of the metabolites will depend on the processes of formation and elimination. However, while administering the same dose of a drug to a group of patients may produce the expected effect in most patients, in some patients it is ineffective and in others, toxic effects are observed. ${ }^{8}$ However, psychotropic drugs can cause clinical manifestations such as intoxications and metabolic alterations, leading to the development of several pathologies, which may complicate the patient's condition during and after psychiatric treatment. ${ }^{1}$

Specifically, the role of serum lipids in the pathophysiology of psychiatric disorders is unclear, although low levels of high density lipoprotein C (HDL-C) and high atherogenic index have been linked 
to these disorders. ${ }^{5}$ In investigating the relationship between serum cholesterol and serotonin metabolism, Steegmans and his team reported that plasma serotonin concentration was low in men with low serum cholesterol, suggesting that serotonin metabolism is implicated in the association between low cholesterol And depression. ${ }^{9}$ Changes in peripheral cholesterol in relation to the central nervous system suggest that the concentration of cholesterol as the main component of the neuronal membrane can determine the availability of serotonin receptors and its transporter, indicating that low serum cholesterol total, decrease the use of cerebral serotonin. ${ }^{6}$ Certainly, the need to provide the psychiatric patient with a better quality of life, has increasingly encouraged the use of psychotropic drugs throughout the world; However, the tendency to present side effects increases the possibility of developing pathologies, which may be related to the metabolism and functions of the lipid components, depending on the appropriate dose titration and medication time. ${ }^{8}$

Patients with mental disorders are at high risk of developing dyslipidemia. It has been shown that specifically antipsychotic and antidepressant drugs activate the transcription factors of the sterol regulatory element binding protein (SREBP) in human and rat glial cells, with the subsequent regulation of numerous genes involved in the biosynthesis of Cholesterol and fatty acids. These factors, which have been shown to increase the biosynthesis of cholesterol in human glial as well as in peripheral cells, may contribute to increased levels of cholesterol in the peripheral blood. In addition, it has been shown that serotonin-norepinephrine reuptake inhibitors alter the regulation of receptors that can affect the control of lipid metabolism, increasing lipolysis in adipose tissue. ${ }^{10,11}$

Myelin contains $70 \%$ lipids in dry weight and cholesterol is an important component among the lipid species found in the myelin sheath. Cholesterol is the essential component in the formation of this substance, its homeostasis being important for the maintenance of myelin structure and its function in the CNS, which is deficient under stress conditions. In turn, under these conditions, the proteinkinase (AMPK- $\alpha$ ) decreases its genetic expression. AMPK- $\alpha$ is a cellular energy sensor that plays an important role in the maintenance of metabolic homeostasis; the phosphorylation of the same causes the phosphorylation of the enzyme Hydroxymethylglutaryl CoA reductase (HMG-CoA reductase), which have a crucial intervention in the synthesis of cholesterol in the brain, because the HMG-CoA reductase, is the limiting enzyme in The synthesis of cholesterol. ${ }^{12}$

\section{Discussion}

All antidepressants have the same efficacy for treatment; however, they differ in adverse effects, which influence patient adherence to treatment, which ultimately translates into clinical effectiveness. It should be noted that many authors point to psychoactive drugs as precursors of obesity, a condition that is closely associated with the hormonal system and lipid metabolism..$^{3,7,8,13}$ Shioiri T et al. ${ }^{14}$ states that there is a direct relationship between the decrease in total cholesterol levels and pharmacological treatment. Le Melledo JM et al. ${ }^{15}$ reported a $9 \%$ increase in LDL-C, which normalized after discontinuation of medication. Martınez Romeroa G et al. ${ }^{16}$ states that treatment with psychopharmaceuticals does not have a significant impact on the prevalence of alterations in lipemia. Paslakis $\mathrm{G}$ et al. ${ }^{17}$ in 2010 found a significant decrease in Lipoprotein A in patients treated with paroxetine but not in those treated with amitriptyline. In 2012 Clayton, AH. And cols found no evident differences in fasting cholesterol levels from baseline to week 52 in treatment. ${ }^{14-18}$

\section{Conclusion}

Psychopharmaceuticals activate the transcription factors of the binding protein to the sterol regulatory elements (SREBP) in glial cells, regulating numerous genes involved in the biosynthesis of cholesterol and fatty acids, which may contribute to increased levels of Cholesterol in the peripheral blood. A situation of sustained stress leads to a decrease in the gene expression of proteinkinase (AMPK- $\alpha$ ) and HMG-CoA reductase, which is reversed with the use of Desvenlafaxacin.

\section{Acknowledgments}

None.

\section{Conflicts of Interset}

None.

\section{References}

1. Acetiuno M, Adán A, Agustí C. Book of health of the clinical hospital of Barcelona and the BBVA Foundation. Editorial Nerea, UK. 2007.

2. Beautrais A, Goldney R, Schlebusch L. Prevention of suicide: an instrument at work. Department of Mental Health and Substance Abuse. World Health Organization, Swizerland, 2006. p. 8

3. Barbui C, Cipriani A, Saxena S. Pharmacological treatment of mental disorders in primary health care. World Health Organization. Washington, USA. 2010.

4. Rodriguez J, Kohn R y Aguilar-Gaxiola S. Epidemiology of mental disorders in Latin America and the Caribbean. Pan American Health Organization. Washington, USA, 2009. p. 632.

5. Londoño N, Marin C, Juarez F, et al. Factores de riesgos psicosociales y ambientales asociados a trastornos mentales. Suma Psicológica. 2009;17(1):59-58.

6. Lüllmann H y Klaus M. Pharmacology text and atlas. Panamericana. Madrid, España. 2010.

7. Chávez E, Ontiveros M, Serrano C. Selective recapture inhibitor antidepressants. Mental health. 2008;31(4):307-319.

8. Martinez G, Peris JF, Gomar J. Use of atypical antipsychotic drugs in institutionalized elderly people and prevalence of metabolic alterations. Hospital pharmacy. 2010;34(3):139-147.

9. Carreto R, Jimenez N, Lozano J. Serum cholesterol concentrations and suicidal intent. Comprehensive medicine from Mexico. 2008;24(3):181185.

10. Raeder MB, Ferno J, Glambek M, et al. Antidepressant drugs activate SREBP and up-regulate cholesterol and fatty acid biosynthesis in human glial cells. Neurosci Lett. 2006;395(3):185-190.

11. Lin HW, Cory AS, Chiung RL, et al. Severe hypertriglyceridemia secondary to venlafaxine use in an older adult on dialysis -case report. BMC Health Services Research. 2007;17:272.

12. Wang J, Qiao J, Zhang Y, et al. Desvenlafaxine prevents white matter injury and improves the decreased phosphorylation of the rate-limiting enzyme of cholesterol synthesis in a chronic mouse model of depression. J Neurochem. 2014;131(2):229-238.

13. Shioiri T, Fujii K, Someya T, et al. Effect of pharmacotherapy on serum cholesterol levels in patients with panic disorder. Acta Psychiatr Scand. 1986;93(3):164-167.

14. Shioiri T, Fujii K, Someya T, et al. Serum cholesterol levels and panic symptoms in patients with panic disorder: a preliminary study. $J$ Affect Disord. 2006;58(2):167-170. 
15. Le Melledo JM, Mailo K, Lara N, et al. Paroxetine-inducedincrease in LDL cholesterollevels. J Psychopharmaco. 2008;1 23(7):826-830.

16. Martinez G, Peris JF, Gomar J. Use of atypical antipsychotic drugs in institutionalized elderly people and prevalence of metabolic alterations. Hospital pharmacy. 2010;34(3):139-147.

17. Paslakis G, Kopf D, Westphal S, et al. Treatment with paroxetine, but not amitriptyline, lowers levels of lipoprotein(a) in patients with major depression. J Psychopharmacol. 2014;25(10):1344-1346.

18. Clayton A, Baker R, Sheehan J, et al. Comparison of adjunctive use of aripiprazole with bupropion or selective serotonin reuptake inhibitors/ serotonin-norepinephrine reuptake inhibitors: analysis of patients beginning adjunctive treatment in a 52 -week, open-label study. $B M C$ Res Notes. 2014;7:459.
19. Florez J, Armijo J, Mediavilla A. Farmacología Humana. 4ta edición, Editorial Masson, Mexico. 2003.

20. Katzung B. Farmacología básica y clinica. (11th edn), Editorial McGrawHill, USA. 2010.

21. Hoyos M, Rosales V. Lípidos: Key Features and Metabolism. Journal of Clinical Update. 2014;41:2142-2145.

22. Ornelas O, Álvarez R, Querales M. Practical Guide to "General Biochemistry”. University of Carabobo, Venezuela, 2014. p. 59-82.

23. Nelson D, Cox M. Lehninger: principios de bioquímica. (5th edn), Editorial Omega, USA. 2009. 